\title{
Capillaria philippinensis
}

National Cancer Institute

\section{Source}

National Cancer Institute. Capillaria philippinensis. NCI Thesaurus. Code C122257.

A species of parasitic nematodes in the family Capillariidae. C. philippinensis causes

intestinal capillariasis. Its life cycle involves fish as intermediate hosts and fish-eating birds as definitive hosts. Humans acquire C. philippinensis by eating infested fish. 